\title{
CD133 mRNA may be a suitable prognostic marker for human breast cancer
}

\author{
Pu Xia \\ Department of Cell Biology, College of Basic Medical Science, Liaoning Medical University, Jinzhou 121001, China \\ Correspondence to: Pu Xia. Department of Cell Biology, College of Basic Medical Science, Liaoning Medical University, Jinzhou 121001, China. \\ Email: nn001007@163.com.
}

\begin{abstract}
Breast cancer is the most commonly malignant cancers in women. CD133 protein is a widely used marker for isolating cancer stem cell (CSC). Its expression is associated with the prognosis of many cancers. However, whether CD133 mRNA could be used as an independent prognostic factor for breast cancer remains inconsistent in previous studies. In this study, I used the Gene Expression-Based Outcome for Breast Cancer Online algorithm (GOBO) software to analysis the prognostic roles of CD133 mRNA in the subtypes of breast cancer. I found that CD133 mRNA is associated with distant metastasis free survival (DMFS) in the patient with all tumors, ER-positive tumors, tamoxifen (TAM) tumors, LN-negative tumors, ER-positive/LN-negative tumors, ER-negative tumors, grade 2 tumors, HER2 enriched tumors, and untreated tumors. These results could provide a molecular target for the subtypes of breast cancer. In this study, I conclude that CD133 mRNA may be a suitable prognostic marker for human breast cancer.
\end{abstract}

Keywords: Breast cancer; CD133; prognosis; Gene Expression-Based Outcome for Breast Cancer Online algorithm (GOBO); bioinformatic analyses

Received: 21 July 2017; Accepted: 17 October 2017; Published: 07 November 2017.

doi: $10.21037 /$ sci.2017.10.03

View this article at: http://dx.doi.org/10.21037/sci.2017.10.03

\section{Introduction}

Breast cancer is the first cause of cancer death in women (1). It is a heterogeneous disease that has been divided into two main types with five subtypes, according to hormone receptor (HR) expression (negative or positive) and epithelial cellular origin (basal or luminal) (2). The HR-negative group has three subtypes: (I) with human epidermal growth factor receptor-2 (HER2) overexpression; (II) normal-like; and (III) basal subtype with positive absent HR (estrogen receptor and progesterone receptor) and absent HER2 expression (triplenegative subtype) (2). The major causes of treatment failure for patients with breast cancer are tumor metastasis (3). Recent years, cancer stem cells (CSCs) are considered to be the origin of tumor metastasis and recurrence (4).

CD133 (prominin-1) is a transmembrane glycoprotein that localizes to membrane protrusions of both cancer cells and normal cells (5). It has been intensively used to isolate CSCs in various solid tumors (6). To our knowledge, the prognostic role of CD133 in breast cancer has not been well established. CD133 expression showed no statistical association with the survival rate of the patients with triplenegative breast cancer (7). However, CD133 expression is an independent poor prognostic factor for invasive breast cancer (8). In this study, we used the Gene ExpressionBased Outcome for Breast Cancer Online algorithm (GOBO, http://co.bmc.lu.se/gobo) that developed by Ringnér and colleagues in Lund University Canceromics Branch (Sweden) (9) to analysis the prognostic roles of CD133 mRNA in the subtypes of breast cancer.

\section{Methods}

GOBO is a publicly available database that including tumor tissue gene expression data and clinical information from 1881 breast cancer subjects. The correlations of CD133 transcript with molecular subtypes, histological grades, and clinical outcomes in breast cancer patients was 

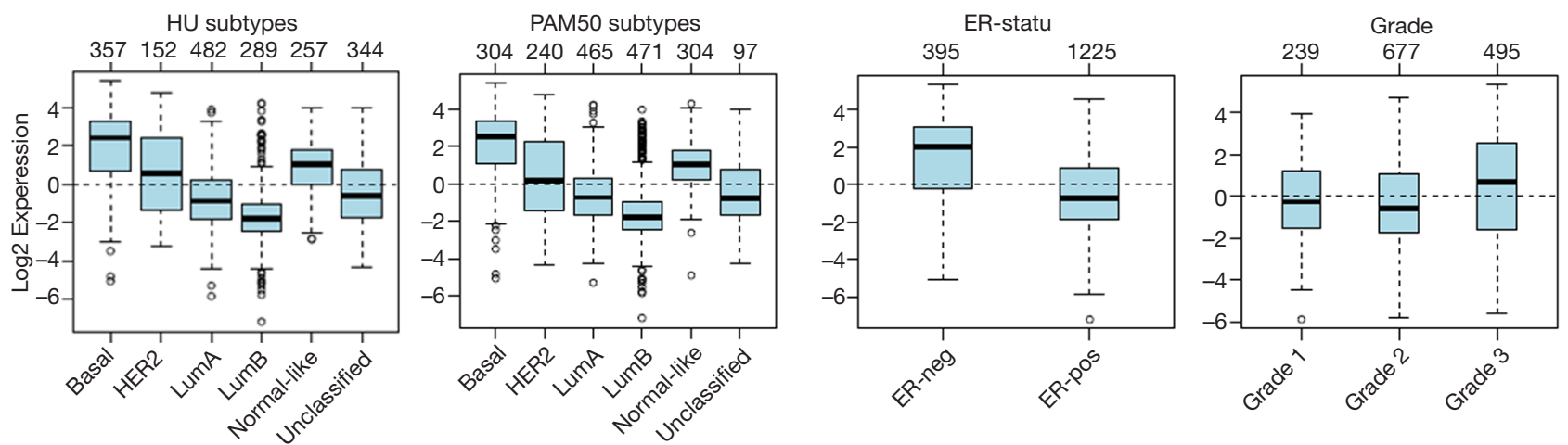

Figure 1 Gene Expression-Based Outcome for Breast Cancer Online algorithm (GOBO) analyses of CD133 transcript among subtypes of breast cancer.

analyzed by using GOBO. The calculated $\mathrm{P}$ values were adjusted for multiple testing by applying a False Discovery Rate adjustment (FDR $=0.2$ ). $\mathrm{P}$ values less than 0.05 were considered statistically significant.

\section{Results}

\section{CD133 mRNA is associated with the survival rate of breast cancer patients}

The highest CD133 mRNA was observed in basal tumors $(\mathrm{P}<0.00001$, Figure 1). ER-negative tumors showed a higher CD133 mRNA than ER-positive ones $(\mathrm{P}<0.00001$, Figure 1$)$. The level of CD133 mRNA was higher in grade 3 tumor than that in grade 1 and grade 2 tumors $(\mathrm{P}<0.00001$, Figure 1$)$. Low CD133 mRNA was a bad prognostic factor for distance metastasis free survival (DMFS) in the patient with all tumors $(\mathrm{P}=1 \mathrm{e}-05)$, ER-positive tumors $(\mathrm{P}=0.005)$, ERBB2 tumors $(\mathrm{P}=0.011)$, tamoxifen (TAM) tumors $(\mathrm{P}=0.004), \mathrm{LN}$ negative tumors $(\mathrm{P}=1 \mathrm{e}-05)$, ER-positive/LN-negative tumors $(\mathrm{P}=0.004)$, ER-negative tumors $(\mathrm{P}=0.008)$, grade 2 tumors $(\mathrm{P}=0.009)$, HER2 enriched tumors $(\mathrm{P}=3 \mathrm{e}-05)$, and untreated tumors $(\mathrm{P}=0.001)$ (Figure S1). High CD133 mRNA is a good indicator for RFS of all tumors $(\mathrm{P}=0.013)$, ER-positive tumors $(\mathrm{P}=0.008)$, HER2 enriched tumors $(\mathrm{P}=0.048)$, and $\mathrm{LN}$-positive tumors $(\mathrm{P}=0.022)$ (Figure 2). After adjustment for age, tumor size, tumor grade, ER-status, and LNstatus, the Kaplan-Meier survival analyses were supported by the corresponding multivariate analyses (Figure 2 and Figure S1).

\section{Discussion}

CD133 protein has been used as a marker to identify
CSC in many solid tumors, including breast cancer (6). CD133 overexpression was significantly correlated with LN metastasis, ER negativity, PR negativity, HER2 positivity, non-luminal subtype, and chemotherapy (8). In this study, we confirmed that that CD133 mRNA is associated with DMFS in the patient with all tumors, ER-positive tumors, ERBB2 tumors, TAM tumors, LN-negative tumors, ER-positive/LN-negative tumors, ER-negative tumors, grade 2 tumors, HER2 enriched tumors and untreated tumors. Many previous studies showed that CD133 protein expression was correlated with a poor survival time of breast cancer $(5,8,10)$. However, breast cancer is a heterogeneous disease. There was often a controversy whether CD133 was a suitable marker for the subtypes of breast cancer. Liu et al. (11) found that CD133 overexpression was associated with poor prognosis of invasive carcinoma. Lin et al. (12) found that CD133 overexpression correlated with negative hormone status and perineural invasion and there was a trend towards correlation with HER2-amplified status in invasive carcinoma. Obviously, the opposite trend of CD133 protein and mRNA was observed in predicting the prognosis of breast cancer. Human CD133 protein is a five transmembrane single-chain glycoprotein that containing two large extracellular and two small intracellular loops (13). The nature of CD133's epitope is a main factor that influenced the antibody binding (14). Both micromilieu and drug treatment could affect CD133 stability and its transport to the cell surface $(15,16)$. We speculate that the different prognostic value may be due to different stem cell characteristics in subtypes of breast cancer. Therefore, the results of the present study require validation via in vivo and in vitro studies. 


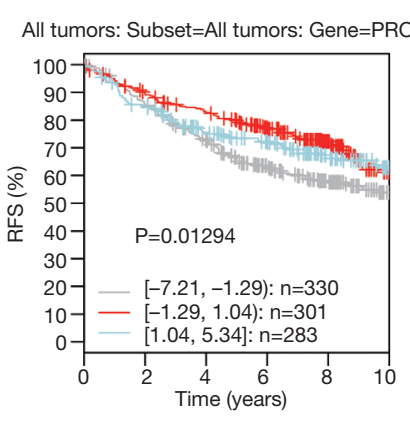

Multivariate analysis for $n=829$ cases

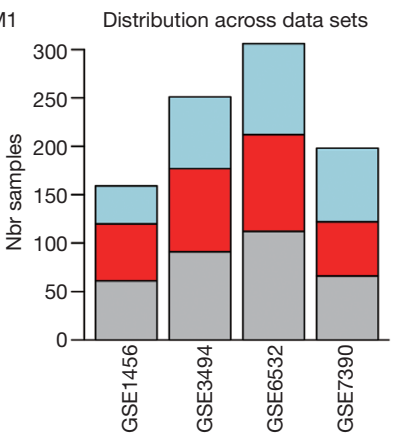

Group sizes multivariate analysis

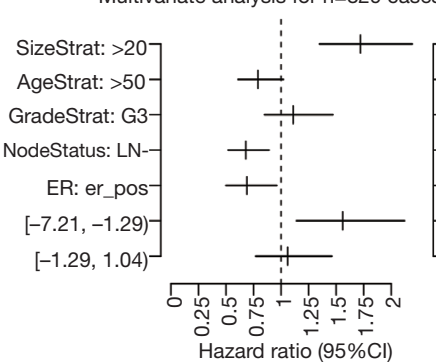

All tumors: Subset=ER-positive: Gene=PROM1

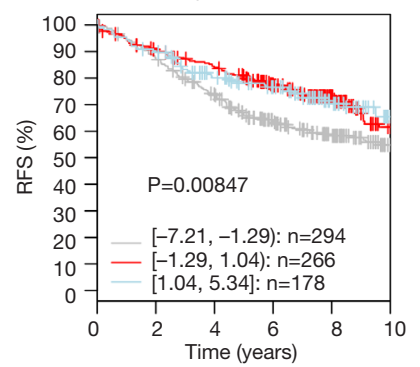

Multivariate analysis for $n=671$ cases

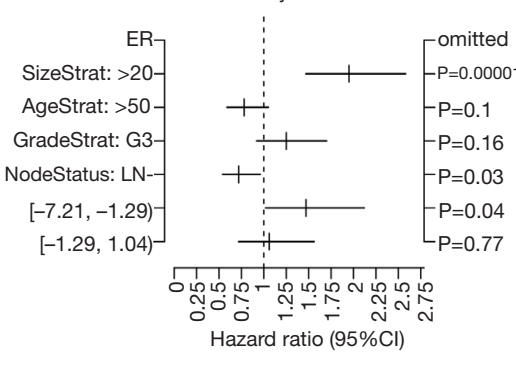

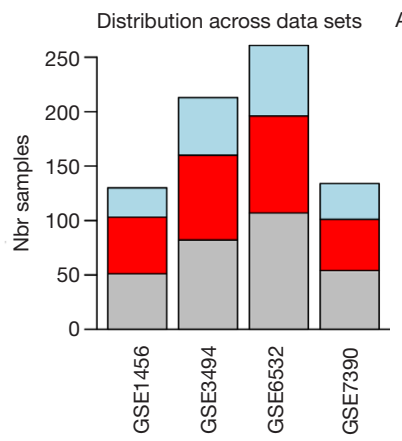

Group sizes multivariate analysis

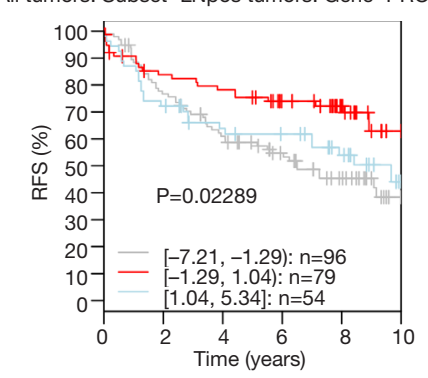

Multivariate analysis for $n=671$ cases

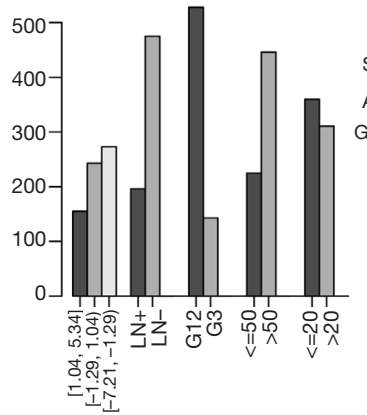

All tumors: Subset=PAM50_HER2enriched tumors: Gene=PROM1
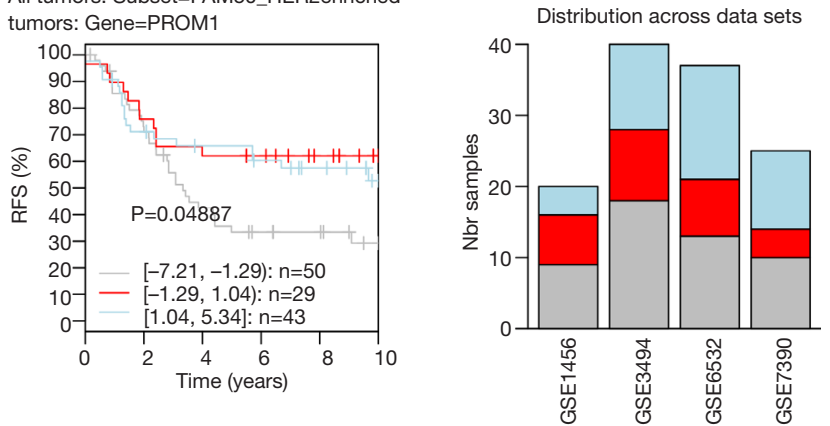

Multivariate analysis for $\mathrm{n}=111$ cases

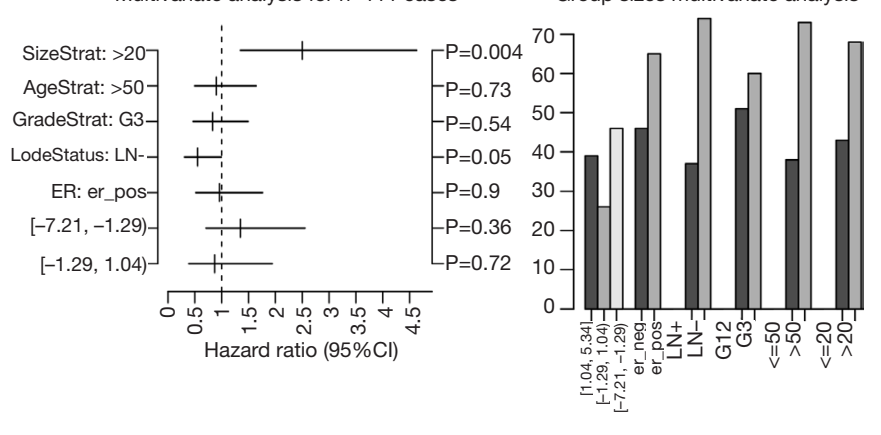

All tumors: Subset=LNpos tumors: Gene=PROM1

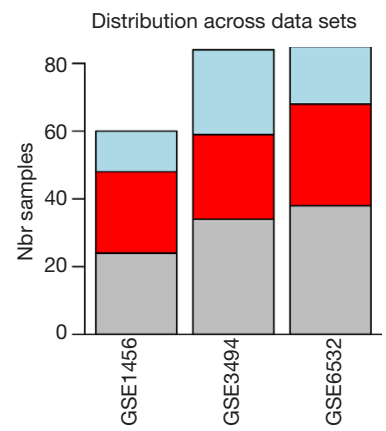

Group sizes multivariate analysis
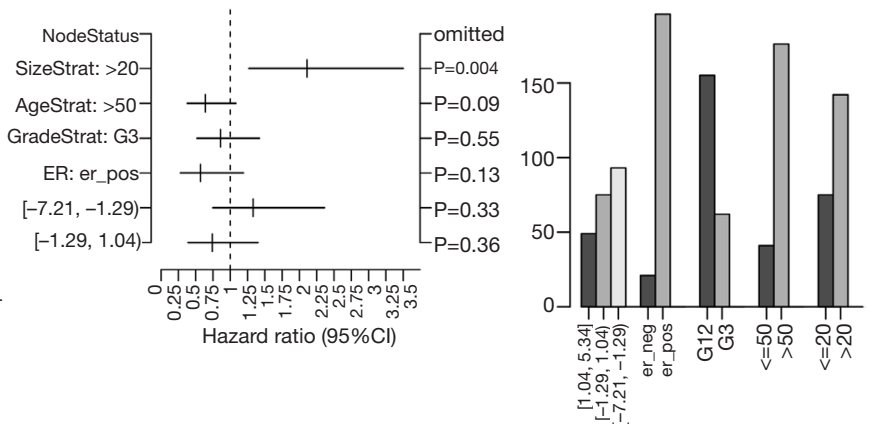

Figure 2 Kaplan Meier analysis of relapse free survival (RFS) in patients with the subtypes of breast cancer expressing high (blue line), intermediate (red line), and low (gray line) CD133 mRNA. 
In summary, based on the results showed above, CD133 mRNA may be suitable for predicting the prognosis of breast cancer, but not CD133 protein. In this study, we provide an overview of prognostic roles of CD133 mRNA in the subtypes of breast cancer. It can provide a choice of molecular target therapy in the clinic.

\section{Acknowledgements}

Funding: This study was supported by National Natural Scientific Foundation of China (No. 81502558) and Talents Introduction Projects of Liaoning Medical University.

\section{Footnote}

Conflicts of Interest: The author has no conflicts of interest to declare.

\section{References}

1. Miller KD, Siegel RL, Lin CC, et al. Cancer treatment and survivorship statistics, 2016. CA Cancer J Clin 2016;66:271-89.

2. Anderson WF, Rosenberg PS, Prat A, et al. How many etiological subtypes of breast cancer: two, three, four, or more? J Natl Cancer Inst 2014;106:dju165.

3. Senkus E, Kyriakides S, Ohno S, et al. Primary breast cancer: ESMO Clinical Practice Guidelines for diagnosis, treatment and follow-up. Ann Oncol 2015;26 Suppl 5:v8-30.

4. Zhang GF, Li CX, Liu ZQ, et al. Cancer stem cell targets - a review. Eur Rev Med Pharmacol Sci 2016;20:2045-51.

5. Schmohl JU, Vallera DA. CD133, Selectively Targeting the Root of Cancer. Toxins (Basel) 2016;8:E165.

6. Xia P. Surface markers of cancer stem cells in solid tumors. Curr Stem Cell Res Ther 2014;9:102-11.

\section{doi: $10.21037 /$ sci.2017.10.03}

Cite this article as: $\mathrm{Xia}$ P. CD133 mRNA may be a suitable prognostic marker for human breast cancer. Stem Cell Investig 2017;4:87.
7. Collina F, Di Bonito M, Li Bergolis V, et al. Prognostic Value of Cancer Stem Cells Markers in Triple-Negative Breast Cancer. Biomed Res Int 2015;2015:158682.

8. Kim SJ, Kim YS, Jang ED, et al. Prognostic Impact and Clinicopathological Correlation of CD133 and ALDH1 Expression in Invasive Breast Cancer. J Breast Cancer 2015;18:347-55.

9. Ringnér M, Fredlund E, Häkkinen J, et al. GOBO: gene expression-based outcome for breast cancer online. PLoS One 2011;6:e17911.

10. Lv X, Wang Y, Song Y, et al. Association between ALDH1+/CD133+ stem-like cells and tumor angiogenesis in invasive ductal breast carcinoma. Oncol Lett 2016;11:1750-6.

11. Liu Q, Li JG, Zheng XY, et al. Expression of CD133, PAX2, ESA, and GPR30 in invasive ductal breast carcinomas. Chin Med J (Engl) 2009;122:2763-9.

12. Lin CH, Liu CH, Wen CH, et al. Differential CD133 expression distinguishes malignant from benign papillary lesions of the breast. Virchows Arch 2015;466:177-84.

13. Fargeas CA, Florek M, Huttner WB, et al. Characterization of prominin-2, a new member of the prominin family of pentaspan membrane glycoproteins. J Biol Chem 2003;278:8586-96.

14. Miraglia S, Godfrey W, Yin AH, et al. A novel fivetransmembrane hematopoietic stem cell antigen: isolation, characterization, and molecular cloning. Blood 1997;90:5013-21.

15. Wu Y, Wu PY. CD133 as a marker for cancer stem cells: progresses and concerns. Stem Cells Dev 2009;18:1127-34.

16. Mak AB, Blakely KM, Williams RA, et al. CD133 protein $\mathrm{N}$-glycosylation processing contributes to cell surface recognition of the primitive cell marker AC133 epitope. J Biol Chem 2011;286:41046-56. 
All tumors: Subset=All tumors:
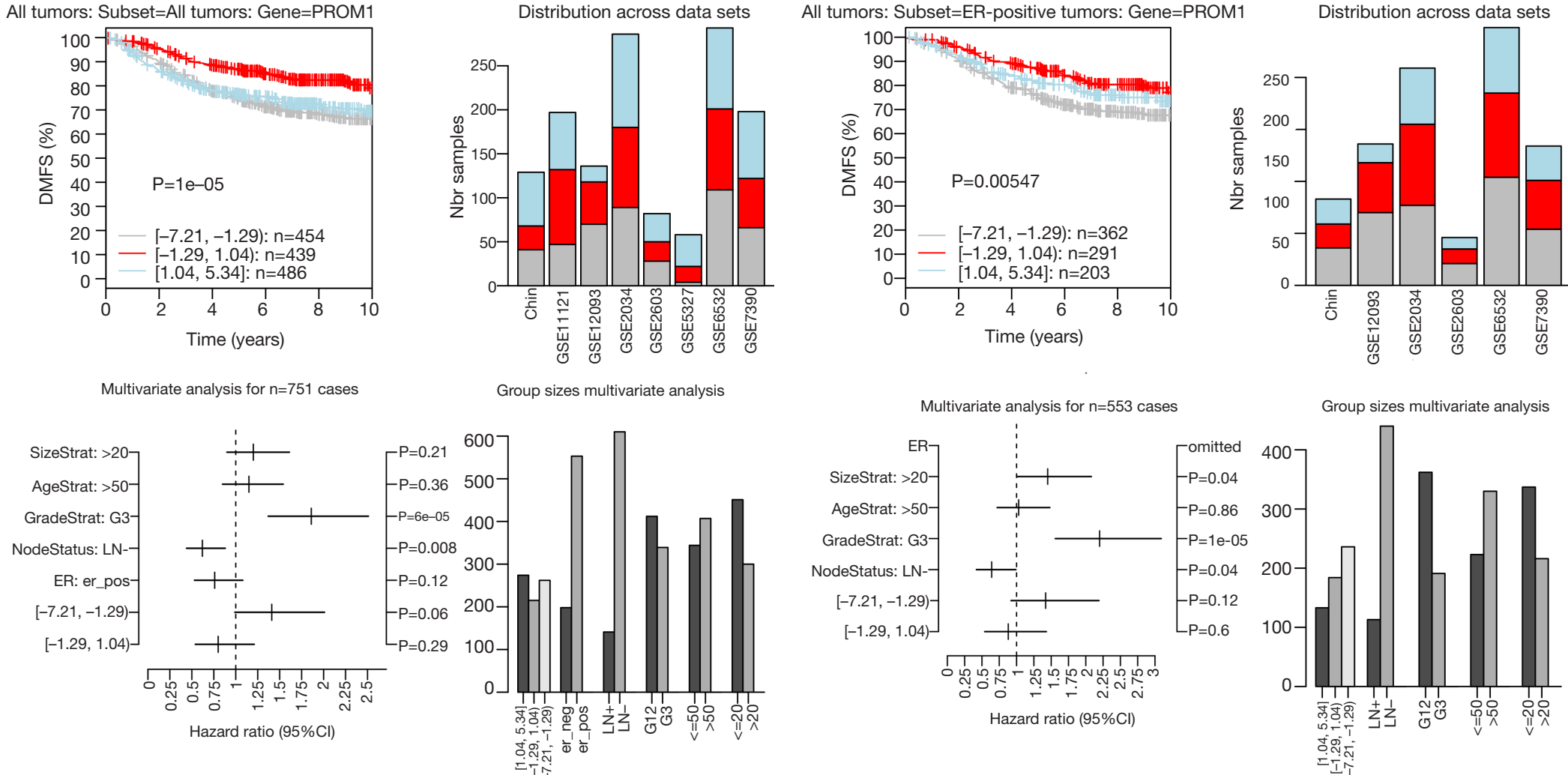

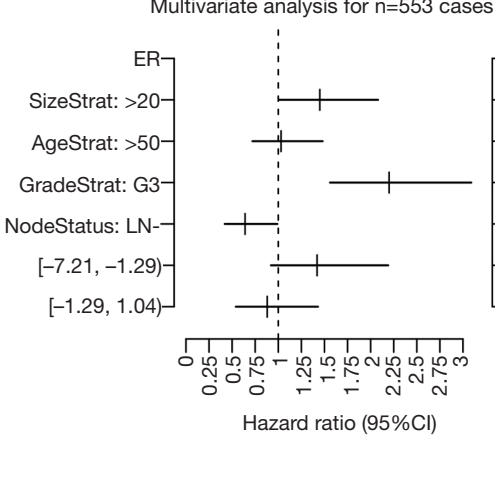
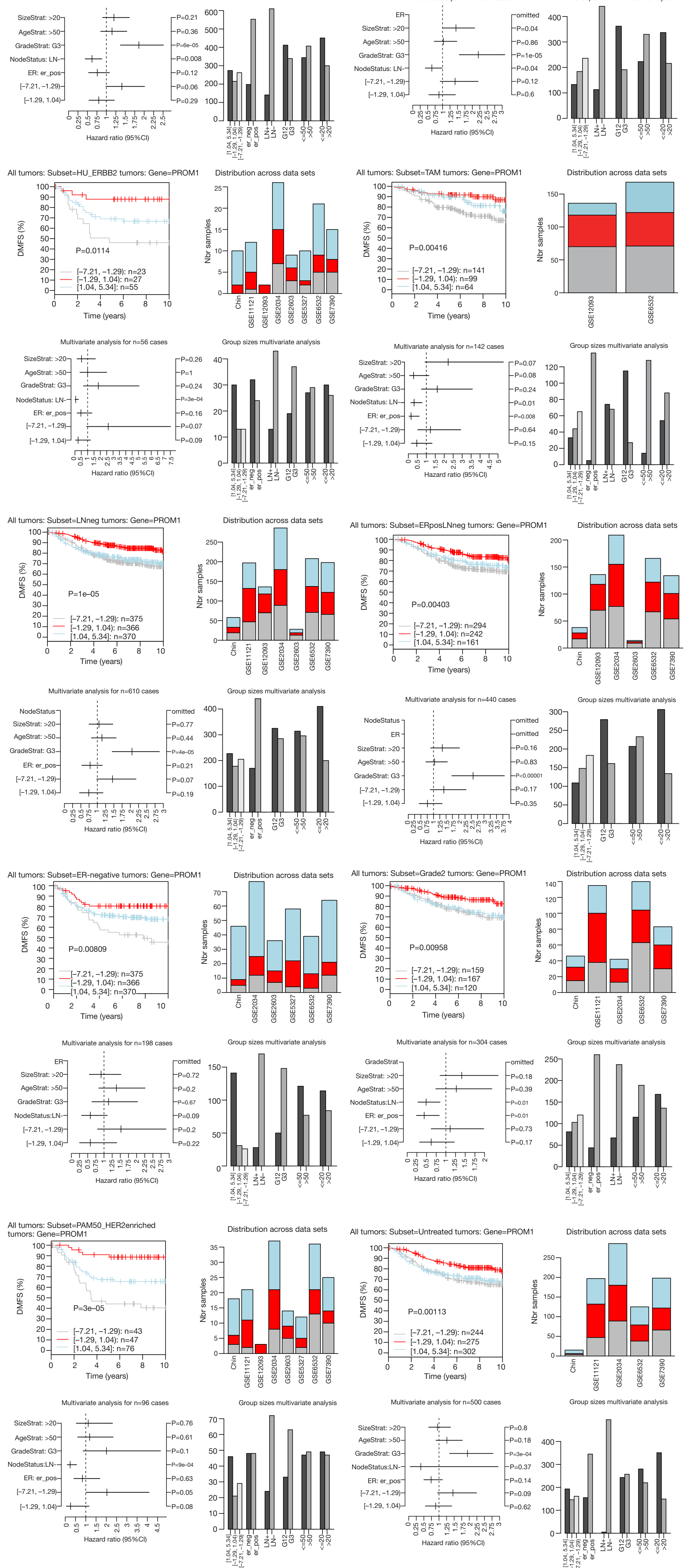